\title{
Evaluación y análisis de propiedades estructurales de productos de madera
}

\section{Evaluation and analysis of structural properties of wood products}

\author{
$\underline{\text { E. Hermoso }}^{(*)}$, R. Mateo ${ }^{(*)}$, J. C. Cabrero ${ }^{(*)}$, J. I. Fernández-Golfín ${ }^{(*)}$
}

\section{RESUMEN}

Los productos de madera destinados a la construcción están dotados de normativa de apoyo para la evaluación de sus propiedades y el control y seguimiento de su calidad. Por las particularidades del material, es necesario disponer de laboratorios especializados que aporten el equipamiento adecuado para la ejecución de dichas tareas y de personal experimentado que valore correctamente los resultados obtenidos.

En este trabajo se presentan los resultados de las evaluaciones desarrolladas durante el año 2012 sobre las propiedades estructurales en distintos productos de madera destinados a la construcción (tableros contrachapados, tableros de partículas, suelos de madera para exterior, madera laminada encolada y madera contralaminada) y se debate sobre los aspectos que afectan a su calidad y adecuación al uso, aportando a la industria las medidas necesarias para la corrección de fallos o la optimización del producto y su diseño, así como proporcionar indicaciones precisas a los usuarios de los productos.

Palabras clave: Ensayos físico-mecánicos; productos de madera estructural; control de calidad; valoración.

\section{ABSTRACT}

Wood products focused on construction are supported by standards for the evaluation of their properties and their quality control. Due to the specific characteristics of these products, specialized laboratories with suitable equipment and qualified people are necessary to value the obtained results.

This paper shows the results of the evaluations conducted during the year 2012 about different structural properties of wood products for construction: plywoods, chipboards, deckings, glued laminated timber, and cross laminated timber. The conditions that affect their quality and fitness for use are discussed, providing to the industry the necessary steps to correct failures or to optimize products and design. Last, precise indications of usefulness are given for the end users of the products.

Keywords: Physical-mechanical tests; structural wooden products; quality control; valuation.

${ }^{(*)}$ Laboratorio de Estructuras de Madera, INIA-CIFOR.

(**) AITIM

Persona de contacto/Corresponding author: hermoso@inia.es (E. Hermoso)

Cómo citar este artículo/Citation: Hermoso, E., Mateo, R., Cabrero, J. C., Fernández-Golfín, J. I. (2015). Evaluación y análisis de propiedades estructurales de productos de madera. Informes de la Construcción, 67(537): eo62, doi: http://dx.doi.org/10.3989/ic.13.061.

Licencia / License: Salvo indicación contraria, todos los contenidos de la edición electrónica de Informes de la Construcción se distribuyen bajo una licencia de uso y distribución Creative Commons Reconocimiento no Comercial 3.o. España (cc-by-nc). 


\section{INTRODUCCIÓN}

El uso de elementos estructurales de madera o sus productos derivados en las construcciones se promueve, en gran parte, mediante el conocimiento de sus propiedades y el control de su calidad. La madera es un producto natural, diferente a los habitualmente empleados en edificación, con especificidades particulares, por eso para alcanzar esos objetivos se requiere de la experiencia de profesionales que sepan ejecutar los ensayos adecuadamente y valorar los resultados obtenidos para transmitirlos por un lado al usuario y por otro al fabricante, contribuyendo de esta forma a la identificación de los factores críticos del material o del diseño y colaborando en su optimización.

Hay que llamar la atención sobre la necesaria periodicidad de los controles para seguimiento tanto de la trazabilidad de la fabricación del producto, como del cumplimiento de los requisitos normativos específicos según el fin al que vayan destinados.

La amplitud de la oferta de mercado que encuentra el usuario del producto a la hora de la elección, le hace receptivo a valorar la información complementaria relativa a las características y el seguimiento realizado sobre el mismo a través de estos controles, principalmente por la orientación a la seguridad adicional que añade en la prescripción y uso.

Complementariamente a los controles voluntarios, como pueden ser los sellos de calidad, existen otras razones motivadas por la reglamentación de obligado cumplimiento común a los elementos constructivos y que por tanto es de aplicación también a los productos de madera.

En julio de 2013 será obligatoria la aplicación del Reglamento de Productos de Construcción 305/2011 (1) en el cual se exige que todo producto de construcción que se encuentre dentro del ámbito de aplicación del Reglamento y que vaya a ser comercializado en la Unión Europea, tenga que llevar, en los plazos determinados, el marcado CE. La orientación del reglamento respecto a la anterior directiva de productos de la construcción ha variado sensiblemente por la desaparición del concepto de idoneidad al uso de los productos con marcado CE, sustituyéndolo por una Declaración de Prestaciones que emite el fabricante pero que supone exclusivamente una expresión de los valores de las prestaciones de las características de su producto, quedando bajo la responsabilidad de la Reglamentación del Estado miembro (2) o, en su defecto, de los técnicos prescriptores del proyecto y/o de la dirección facultativa, los valores que garanticen la idoneidad del producto para la obra concreta.

Así pues, para comprobar las prestaciones del producto se deben utilizar los medios y herramientas definidos en la legislación (3) como los controles y los ensayos de recepción de producto.

El Reglamento define «producto de construcción» como cualquier producto o kit fabricado e introducido en el mercado para su incorporación con carácter permanente en las obras de construcción o partes de las mismas y cuyas prestaciones influyan en las prestaciones de las obras de construcción en cuanto a los requisitos básicos de tales obras. Entre los puntos principales que enumera el Anexo I del citado Reglamento en cuanto a requisitos básicos, se encuentra la resistencia mecánica y estabilidad, que es la que nos compete en este artículo.

El Laboratorio de Maderas del INIA-CIFOR está acreditado por ENAC según ISO 17025 para la ejecución de ensayos en este ámbito pero además dispone de equipamiento apropiado y personal cualificado para realizar otros ensayos acordes con normativa existente y con protocolos particulares solicitados por los clientes y asesorados por los técnicos del laboratorio. Adicionalmente, el Laboratorio es Organismo Notificado $\left(\mathrm{n}^{\circ}\right.$ 1551) para la realización de trabajos de evaluación de productos de la familia de los «Tableros de madera» según la norma EN 789 (4) (ensayo físico-mecánico).

Acorde a lo expuesto, la industria requiere de este tipo de ensayos no solamente para cumplir la legislación vigente, sino también para conocimiento de los productos que fabrica y para el desarrollo industrial de nuevos productos que lanzar al mercado.

El objetivo de este artículo es presentar los trabajos realizados durante el año 2012 en el Laboratorio de Maderas del INIA-CIFOR como ejemplo del control que se realiza sobre los productos de madera y la utilidad de los resultados generados para al sector (fabricantes y usuarios).

\section{CARACTERÍSTICAS EVALUADAS SOBRE LOS PRODUCTOS DE MADERA}

\subsection{Resistencia al punzonamiento en tableros contrachapados estructurales}

Los tableros contrachapados son productos que se obtienen encolando varias chapas de madera desenrolladas del tronco de tal manera que las fibras de dos chapas consecutivas formen entre sí un ángulo de 90 grados. Son muy resistentes y relativamente indeformables ya que las chapas contiguas en distintas direcciones hacen que se compensen las tensiones internas del material. Se pueden fabricar con diversas maderas: Pino radiata, Chopo, Eucalipto, Calabó, Okume, etc. y distintos tipos de adhesivos según el uso (interior o exterior). Algunos tableros se construyen de forma mixta para optimizar determinadas propiedades, es decir, combinando diversos tipos de madera.

Sus aplicaciones son muy diversas pudiendo encontrarse en encofrados, en estructuras tanto exteriores como interiores formando parte de bases de cubiertas de techo y forjado, divisiones o utilizarse para construcción de perfiles y paneles estructurales. Además pueden ser empleados en revestimientos tanto de pilares, vigas, muros o tabiques y con diferentes acabados como estucado, pintado o barnizado según los requerimientos de los usuarios.

Son aptos para diversos recubrimientos exteriores, como por ejemplo papel fenólico que aporta resistencia a la abrasión y al desgaste y permite varias reutilizaciones, o chapas metálicas para su aplicación en suelos de vehículos industriales, que es el caso que seguidamente trataremos. Según su destino las especificaciones mecánicas del producto serán diferentes.

Para el uso en suelos de vehículos industriales el tablero necesita un acabado antideslizante que permita el tránsito por él sin riesgo. Su evaluación requiere reproducir el efecto de la carga concentrada en la rueda de un traspalé, lo que se consi- 
gue mediante la simulación de un punzonamiento mediante la aplicación de una carga puntual de compresión.

El acabado antideslizante de los tableros ensayados corresponde a una chapa metálica de dos texturas diferentes (Figura 1). Las dimensiones de estos tableros son $24 \times 380 \times 900 \mathrm{~mm}^{3}$.

La evaluación de la rotura del tablero en este ensayo no resulta sencilla ya que no se produce una caída de carga ni una rotura brusca, sino que se aprecian descensos de carga sucesivas correspondientes a las roturas de las diferentes capas. Es necesario conocer el producto para detectar el momento real en el que ha perdido su capacidad resistente.

Los valores medios de los resultados obtenidos aparecen en la Tabla 1.

En este caso la comparación de los resultados obtenidos con los de expedientes anteriores sobre el mismo tipo de producto nos permite concluir que la calidad del producto no ha disminuido. Como cabe esperar, además de su finalidad antideslizante, la presencia de la chapa metálica en la capa exterior se traduce en un significativo aumento en la capacidad de carga del tablero ya que en ensayos similares efectuados con idéntica metodología sobre tableros soporte similares pero sin acabado alguno, se obtuvieron valores de resistencia al punzonamiento casi tres veces inferiores, resistencia que se vio reducida a la mitad en los productos que incorporaban una capa de acabado compuesta de fibra de vidrio.

En este caso el tablero es un soporte ideal por su ligereza y su capacidad portante para transmitir las cargas de flexión hacia la estructura de soporte, a la vez que coadyuva a mejorar la resistencia de la capa superficial. Por dicho motivo su uso está ampliamente extendido en la construcción de plataformas de carga de vehículos industriales, medios de transporte (autobuses urbanos e interurbanos, trenes) y embalajes especiales.

\subsection{Propiedades indicadoras del cálculo de tableros estructurales de partículas}

Los tableros de partículas son productos obtenidos aplicando presión y calor sobre una mezcla compuesta de partículas de madera y/o otros materiales lignocelulósicos y diversos tipos de adhesivo y aditivos, elegidos todos ellos en función del destino del tablero, de esta forma se pueden obtener productos con propiedades mejoradas tales como la repelencia a la humedad (tableros hidrófugos), el comportamiento frente al fuego (tableros ignífugos) o la resistencia frente a organismos xilófagos (5).

Los tableros de partículas suelen ser habituales no solo en productos de carpintería sino también en aplicaciones constructivas como bases de cubiertas, de forjados, en viguetas mixtas, encofrados, tableros sándwich, etc., siendo requisito en estos últimos que los tableros pertenezcan a las «clases técnicas» de tableros estructurales. Se definen cuatro clases técnicas de tableros estructurales según su uso (6):

- Tablero P4, para su utilización en ambiente seco.

- Tablero P5, para su utilización en ambiente húmedo.

- Tablero P6 de alta prestación para su utilización en ambiento seco.

- Tablero P7 de alta prestación para su uso en ambiente húmedo.

Tabla 1. Resultados de los ensayos de punzonamiento.

\begin{tabular}{|l|c|c|c|c|c|}
\hline Acabado & $\begin{array}{c}\text { Espesor } \\
(\mathbf{m m})\end{array}$ & $\begin{array}{c}\text { Cara } \\
(\mathbf{m m})\end{array}$ & $\begin{array}{c}\text { Long. } \\
\mathbf{( m m )}\end{array}$ & $\begin{array}{c}\text { Valor medio } \\
\text { Humedad } \\
\mathbf{( \% )}\end{array}$ & $\begin{array}{c}\text { Valor medio de } \\
\text { Fuerza (kN) }\end{array}$ \\
\hline Tipo 1 & 28 & 380 & 900 & 11,7 & $90,58 \pm 0,23$ \\
\hline Tipo 2 & 29 & 380 & 900 & 11,4 & $92,68 \pm 0,25$ \\
\hline
\end{tabular}
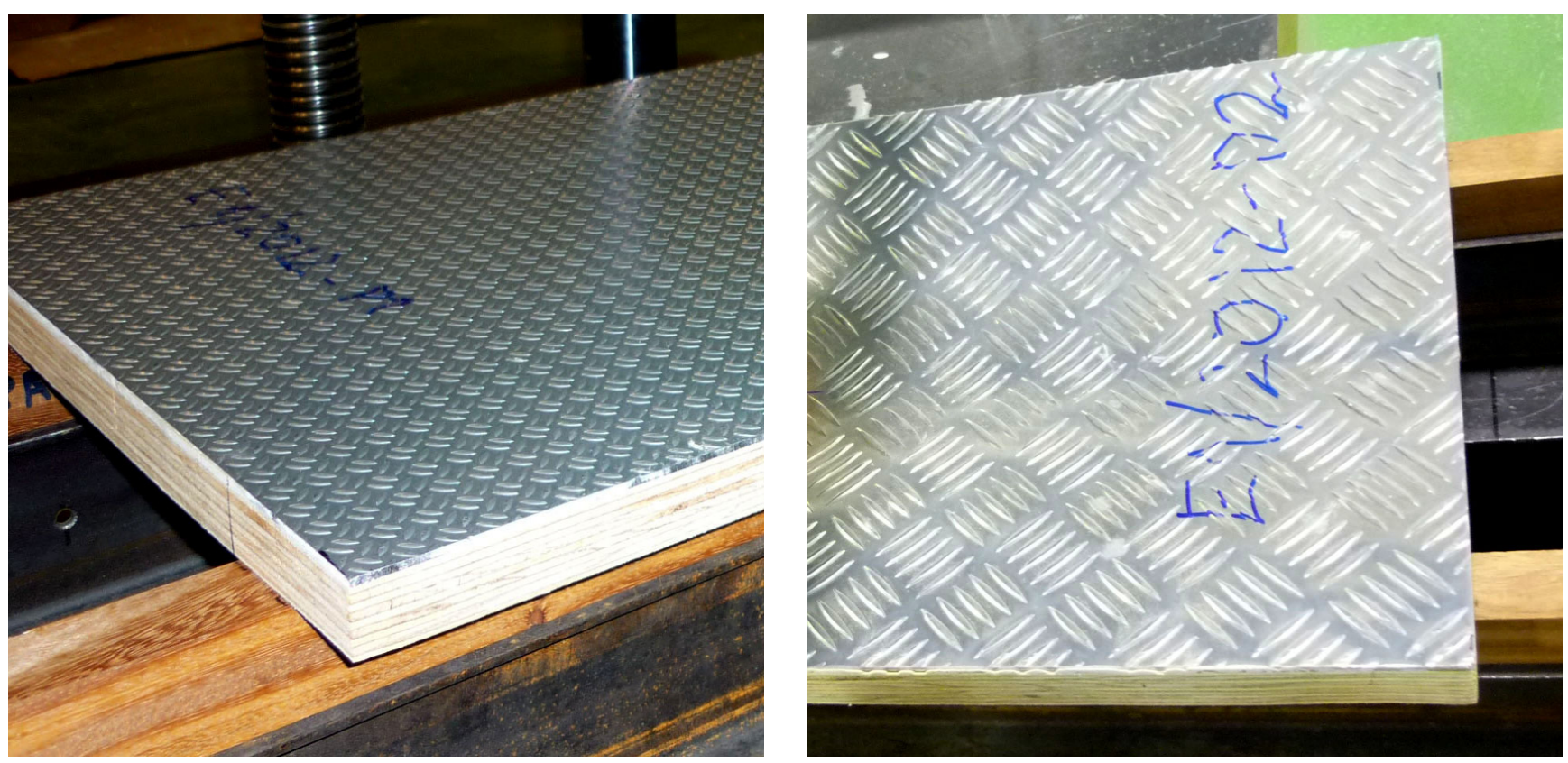

Figura 1. Tableros contrachapados antideslizantes. Acabado tipo 1 a la izquierda, tipo 2 a la derecha. 
Los valores de las propiedades mecánicas de estos tableros, a diferencia de los no estructurales, están caracterizados conforme a la norma armonizada (7).

Los resultados presentados corresponden a los últimos trabajos realizados sobre los tableros estructurales de los tipos P4, P5 y P6 con distintos espesores que van desde $16 \mathrm{~mm}$ hasta $40 \mathrm{~mm}$. La anchura de los tableros es de $300 \mathrm{~mm}$ y su longitud está entre 1,10 m y 1,25 m, dependiendo del tipo de tablero de que se trate.

\subsubsection{Evaluación de las propiedades de flexión}

La norma UNE-EN 789 establece el procedimiento metodológico a seguir para la caracterización físico-mecánica de los tableros estructurales y por ello aporta indicaciones sobre cómo llevar a cabo el acondicionado de la muestra, la medida de las dimensiones, disposición del ensayo (Figura 2), la velocidad del ensayo para controlar los fenómenos de fluencia y el número de probetas necesario por tipo y espesor de tablero (256 probetas en este estudio).

En la Figura 3 se presenta, de forma gráfica usando diagramas de barras, el resumen de los resultados obtenidos para los tipos de tableros $\mathrm{P}_{4}, \mathrm{P}_{5}$ y $\mathrm{P} 6$.

Tras el análisis de los resultados, en líneas generales se observa que la resistencia de los tableros aumenta del tipo P4 al P6 independientemente del espesor, tal y como era de esperar al ser estos últimos (P6) tableros de alta prestación. Los valores obtenidos superan los mínimos exigidos por la norma de referencia, la UNE-EN 12369-1 (Tabla 2).

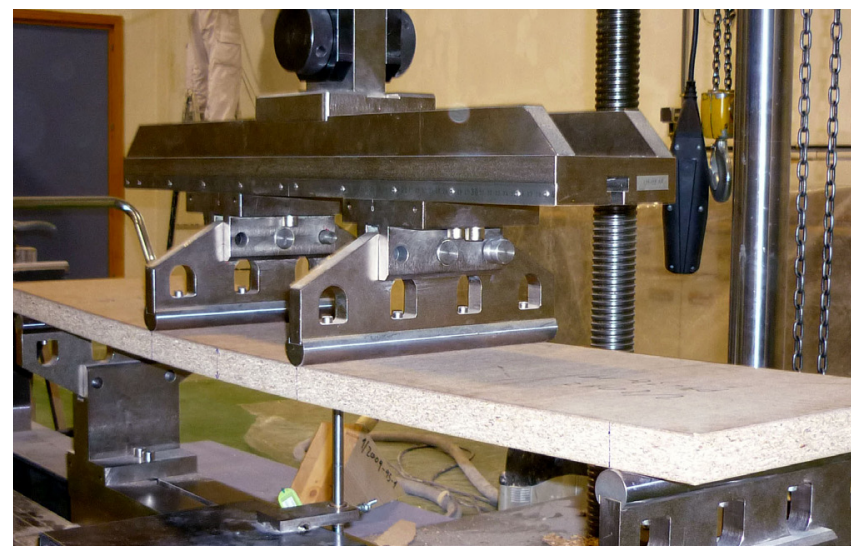

Figura 2. Ensayo de flexión en tablero de partículas.

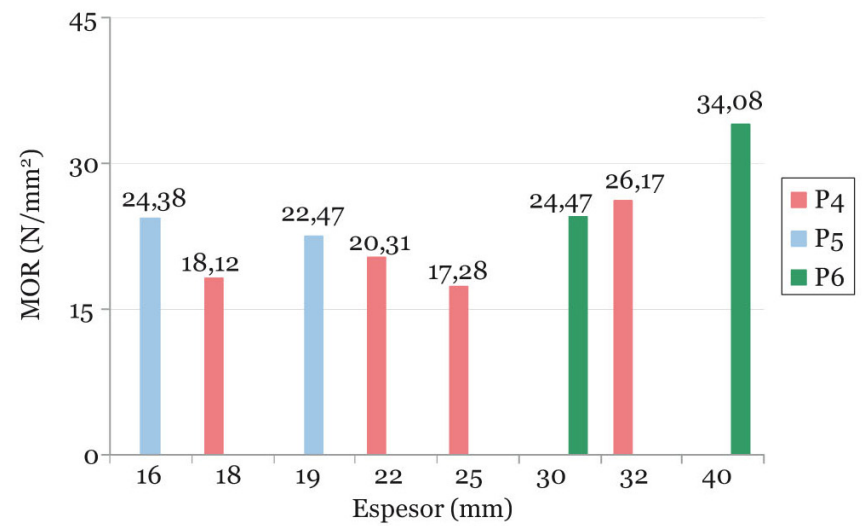

En el módulo de elasticidad se observa una tendencia a presentar valores superiores en los tipos con espesores más bajos (P5: 16, $19 \mathrm{~mm})$ respecto a los más altos (P6: 30, $40 \mathrm{~mm})$.

Un beneficio de realizar este tipo de evaluación sobre los distintos tipos de tableros radica en que el fabricante puede aportar al prescriptor los valores obtenidos para su producto concreto y ser usados para el cálculo en sustitución de los aportados por la normativa de forma generalista.

Tabla 2. Especificaciones de las propiedades mecánicas de los tableros de partículas según UNE-EN 12369-1.

\begin{tabular}{|c|c|c|c|}
\hline Tablero & Espesor (mm) & $\operatorname{MOR}\left(\mathrm{N} / \mathrm{mm}^{2}\right)$ & $\operatorname{MOE}\left(\mathrm{N} / \mathrm{mm}^{2}\right)$ \\
\hline \multirow{4}{*}{$\mathrm{P}_{4}$} & 18 & 12,5 & 2900 \\
\hline & 22 & 10,8 & 2700 \\
\hline & 25 & 10,8 & 2700 \\
\hline & 32 & 9,2 & 2400 \\
\hline \multirow{2}{*}{$\mathrm{P}_{5}$} & 16 & 13,3 & 3300 \\
\hline & 19 & 13,3 & 3300 \\
\hline \multirow{2}{*}{ P6 } & 30 & 12,5 & 3300 \\
\hline & 40 & 11,7 & 3100 \\
\hline
\end{tabular}

\subsubsection{Evaluación de la densidad}

Una de las ventajas determinantes del uso de los productos de madera en la construcción es la buena relación resistencia-peso que aportan, es decir, coadyuvan a la reducción del peso de la estructura en su conjunto (con el correspondiente efecto en el dimensionamiento de las cimentaciones y de los elementos principales de la estructura) sin menoscabo de la resistencia.

Para garantizar la repetitibilidad y la posibilidad de intercomparación de los resultados, este ensayo se realiza conforme a lo definido en normativa (8).

Las probetas utilizadas son 36 , todas de $50 \times 50 \mathrm{~mm}$ y con el espesor correspondiente al tablero, escogidas de 6 tableros de forma aleatoria elegidos entre 18.

Los datos de densidad resultantes de los tableros de partículas se muestran en la Figura 4.

Comparando con los valores especificados en la norma UNEEN 12369-1 para estos tableros (Tabla 3) se deriva que los valores obtenidos en el ensayo son superiores a los exigidos,

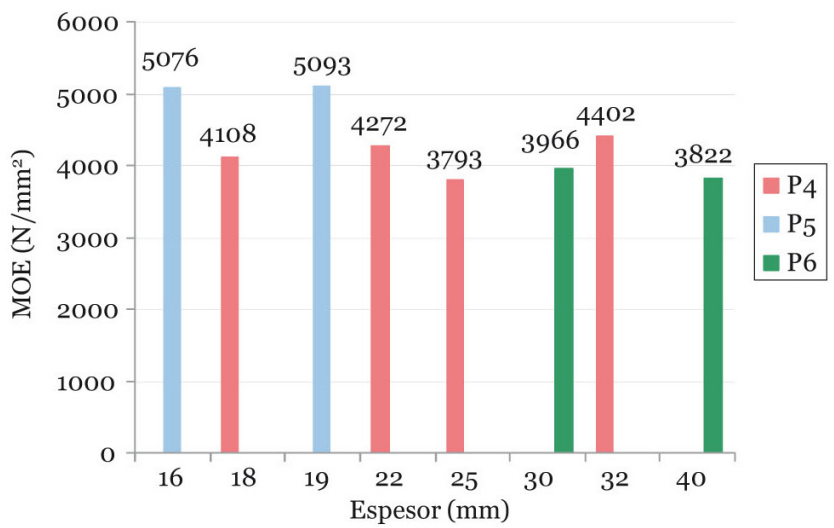

Figura 3. Resultados de la resistencia y la rigidez a la flexión. 


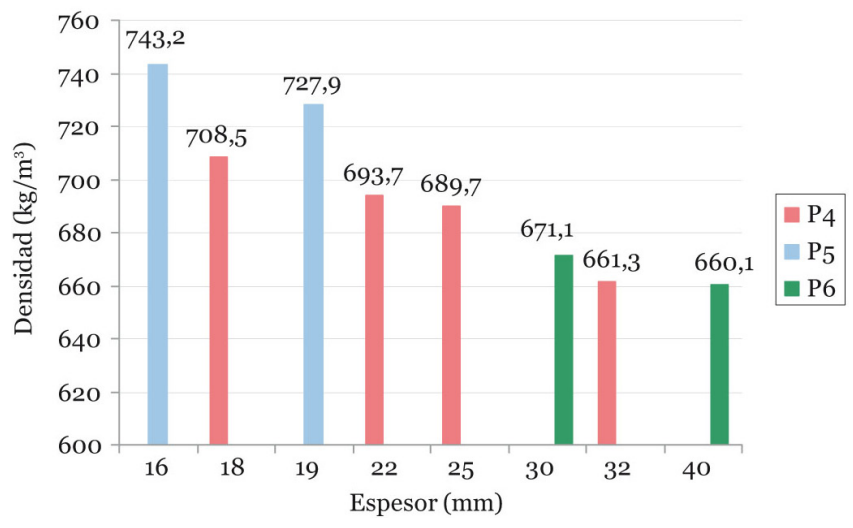

Figura 4. Resultados de la densidad de tableros de partículas.

Tabla 3. Especificaciones de la densidad para tableros de partículas según UNE-EN 12369-1.

\begin{tabular}{|c|c|c|}
\hline Tablero & Espesor $(\mathbf{m m})$ & Densidad $\left.\mathbf{( k g} / \mathbf{m}^{3}\right)$ \\
\hline \multirow{4}{*}{ P4 } & 18 & 600 \\
\cline { 2 - 3 } & 22 & 550 \\
\cline { 2 - 3 } & 25 & 550 \\
\cline { 2 - 3 } & 32 & 550 \\
\hline \multirow{2}{*}{ P5 } & 16 & 600 \\
\cline { 2 - 3 } & 19 & 600 \\
\hline \multirow{2}{*}{ P6 } & 30 & 550 \\
\cline { 2 - 3 } & 40 & 500 \\
\hline
\end{tabular}

siguiendo la tendencia de presentar densidades decrecientes conforme aumenta el espesor.

Los resultados obtenidos permiten asegurar que los tableros objeto de evaluación tendrán un comportamiento superior a la media en aplicaciones estructurales siempre y cuando sean empleados en las condiciones de servicio adecuadas a su clase técnica.

\subsection{Resistencia de las uniones en suelos de madera para exterior}

Este apartado se refiere a los pavimentos de madera en distintos formatos que se colocan al exterior, tanto bajo cubierta como completamente expuestos. Consiste en un entarimado donde las lamas se separan por el canto para dejar pasar el agua de lluvia. Normalmente está indicado en lugares donde se desea dar un tacto más confortable, como terrazas, cenadores, patios, y sobre todo en espacios acuáticos donde se pueda andar descalzo, como solariums de piscinas o paseos marítimos (9).

La tarima puede ser de materiales diferentes. En este caso es de madera maciza, la cual debe tener una durabilidad natural suficiente y si no es así, deberá aplicarse un tratamiento adecuado para que se alcance.

El producto ensayado de dimensiones $20 \times 100 \times 400 \mathrm{~mm}^{3}$ consta de tablas de madera maciza unidas longitudinalmente mediante unión dentada. Se evalúa la resistencia a flexión de dicha unión sobre dos lotes, uno de ellos al que no se le aplica ningún tratamiento previo al ensayo y el segundo al cual se le somete a un proceso de envejecimiento acelerado de aplicación a los adhesivos D4 de carpintería exterior.

La forma y características de la rotura de las uniones dentadas tras ensayo mecánico es analizada en detalle para detectar si existen fallos de encolado o de otro tipo que pudieran haber influido en el resultado.

Los valores medios de la tensión de rotura alcanzados en los dos lotes se presentan en la Tabla 4.

En este caso, a la vista de los resultados, se puede derivar que el tratamiento de envejecimiento no altera las propiedades resistentes de la unión del material, determinante en el uso exterior al que va destinado.

El hecho de que el envejecimiento aplicado no haya degradado mecánicamente la unión permite deducir que tanto el tipo de cola empleado como el propio proceso de aplicación de la misma han sido correctos y que, por tanto, la tarima ensayada puede ser empleada en condiciones de exterior siempre y cuando la madera elegida posea o durabilidad natural o durabilidad conferida suficientes.

\subsection{Control de la madera laminada encolada}

La madera laminada se obtiene por medio del encolado de láminas de madera aserrada de espesor menor de $45 \mathrm{~mm}$, permaneciendo las fibras orientadas de forma paralela a la directriz de la pieza. Se utiliza principalmente para la fabricación de elementos estructurales de grandes longitudes y secciones adaptables a las exigencias arquitectónicas y de ingeniería. La dificultad de obtener piezas de grandes dimensiones de madera aserrada, libre de defectos, hace de la madera laminada un excelente producto para la construcción. Además, la resistencia de la madera laminada puede ser hasta un $30 \%$ mayor que la madera maciza de las mismas dimensiones.

Es muy común encontrar madera laminada como elemento constructivo en estructuras de cubierta, pérgolas, cubiertas de recintos públicos con grandes luces, en construcciones de arcos, elementos de sección variable, y estructuras de diseños complejos, donde cada vez son más comunes.

Dentro de este capítulo, y debido a que la tipología de ensayos es común a ambos, también se incluyen los productos denominados Dúos, basados en el mismo concepto que la madera laminada pero no reconocidos como tales en el CTE DB SEM. Se componen solamente por dos láminas pero de espesores superiores a $45 \mathrm{~mm}$. El uso habitual de éstos es en elementos secundarios de estructuras de grandes luces, en estructuras principales en viviendas, en estructuras de cubierta de viviendas residenciales, etc. (10).

La unión encolada, al igual que ocurre con otros productos encolados, no supone ningún problema desde el punto de vis-

Tabla 4. Resultados del ensayo de flexión de la unión dentada.

\begin{tabular}{|c|c|c|}
\hline & \multicolumn{2}{|c|}{ Tensión de fallo } \\
\hline & Valor medio $\left(\mathrm{N} / \mathbf{m m}^{2}\right)$ & Desviación típica $\left(\mathrm{N} / \mathbf{m m}^{2}\right)$ \\
\hline Lote 1: Probetas sin tratamiento & $28,01 \pm 0,12$ & 5,18 \\
\hline Lote 2: Probetas con tratamiento & $28,08 \pm 0,12$ & 4,12 \\
\hline
\end{tabular}


ta mecánico siempre que se asegure que las uniones resisten al menos lo mismo que la madera, para lo cual deben ser establecidos en fábrica sistema internos de control de la producción, avalados por los correspondientes certificados que así lo indiquen. El hecho de que gran parte de estos elementos realizan funciones estructurales de importancia y que, por tanto, el Marcado CE de obligada presencia en la gran mayoría de productos, establece para ellos los más estrictos Sistemas de evaluación y verificación de la constancia de la prestaciones (típicamente el 1), hace que la calidad venga en cierto modo asegurada por evaluaciones periódicas llevadas a cabo por Laboratorios acreditados. Ejemplos de tales evaluaciones son los que seguidamente presentamos.

\subsubsection{Valoración de la calidad de encolado de las líneas de adhesivo}

Con este ensayo se pretende analizar el encolado entre las láminas de madera en sentido transversal a la pieza mediante la simulación de un envejecimiento acelerado producido en autoclave.

Se definen tres métodos en la norma de aplicación (11) que recoge las especificaciones y requisitos de fabricación de la madera laminada encolada. La elección de cada uno depende de la clase de uso a la que esté destinado el material. En la Tabla 5 se presentan las especificaciones normativas permitidas de los valores máximos del porcentaje de delaminación. En algunos métodos se establece la realización de varios ciclos en caso de incumplimiento.

Tabla 5. Valores del porcentaje máximo de delaminación permitido según el método y el número de ciclos.

\begin{tabular}{|c|c|c|c|}
\hline \multirow{2}{*}{ Método } & \multicolumn{3}{|c|}{ Número de ciclos } \\
\cline { 2 - 4 } & $\mathbf{1}$ & $\mathbf{2}$ & $\mathbf{3}$ \\
\hline A & - & $5 \%$ & $10 \%$ \\
\hline B & $4 \%$ & $8 \%$ & - \\
\hline C & $10 \%$ & - & - \\
\hline
\end{tabular}

Durante el último año se ensayaron en el laboratorio 18 muestras, 16 por el método B y 2 por el método C (Figura 5). El resultado total analizado no superó las especificaciones en un $36 \%$ de las piezas ensayadas. Las causas fueron variadas, pudiendo deberse a la presión insuficiente aplicada en el proceso del fraguado, a la deficiencia de adhesivo recibida en alguna

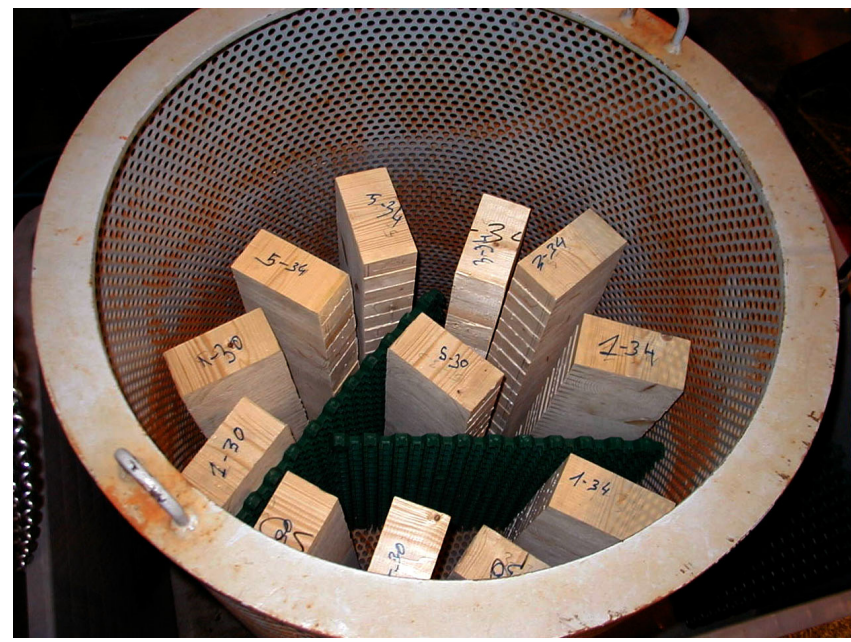

Figura 5. Ensayo de delaminación. Probetas en autoclave. lámina por obturación puntual de los inyectores, a tiempos de espera excesivos para la cola en la constitución del material, a la incorrecta planitud de las láminas o a condiciones ambientales inapropiadas durante el proceso de encolado.

Los controles sucesivos periódicos sobre el material permitirán valorar si las medidas adoptadas para la resolución son efectivas.

La evaluación de la calidad del encolado en laboratorio suele ser una prueba muy extrema ya que en muchas ocasiones, para no perder dinero, los fabricantes toman las muestras de ensayo de los extremos de las piezas, que son donde más fácilmente pueden presentarse problemas de calidad. Cuando esto ocurre, es necesario tomar contramuestras de la parte central, lo que genera notables gastos al fabricante pero aporta una enorme seguridad sobre la calidad real del encolado. Es obvio que si la evaluación sobre estas piezas extremas es correcta, con más motivo lo será la de las partes centrales. También es necesario apuntar que a veces se detectan fallos puntuales debido a la presencia de pequeñas particularidades como bolsas de resina, nudos, etc. En general puede afirmarse que cuando un material está incorrectamente encolado aporta resultados no conformes en casi todas sus líneas de cola y probetas.

\subsubsection{Evaluación de los empalmes de unión dentada}

En sentido longitudinal la madera laminada está constituida por tablas empalmadas mediante uniones dentadas. La geometría y especificidades de fabricación vienen definidas por normativa debiendo cumplir tal unión de forma individual, un mínimo de resistencia a la flexión (12) para considerarse apta para los fines estructurales del producto.

El fabricante debe tener declarado un valor de resistencia para cada una de sus líneas de producción. El muestreo y ensayo tienen como objetivo evaluar la resistencia característica de la unión encolada en las distintas líneas de producción, los diferentes turnos de trabajo o cualquier otra variable que pueda influir y se deba tener en cuenta, la cual no debe ser menor que la resultante de multiplicar un factor estadístico $\left(\mathrm{k}_{15}\right)$, aportado en la norma (13) por el valor declarado por el fabricante.

En la Tabla 6 se presentan los resultados medios de la resistencia a la flexión realizados en el último año sobre 54 muestras (18 por fabricante), correspondientes a tres fabricantes (A, B y C).

Se deriva de los datos obtenidos que la calidad de la madera laminada evaluada en los expedientes de los fabricantes A y B es similar con valores medios sin diferencias significativas, resultando $\mathrm{C}$ los valores más altos. Además se observan variaciones en la desviación típica, de forma que el fabricante $\mathrm{C}$ que obtiene el valor medio más alto, también tiene una dispersión de resultados superior.

Para completar el estudio y aportar una mejor información sobre las causas del fallo, se evalúa el porcentaje de fallo por madera producido en la rotura de la unión de cada pieza ensayada. Cuanto más próximo al 100 \% sea el resultado de la evaluación, mejor encolado pondrá de manifiesto ya que la situación perfecta es aquella en la que la unión falla totalmente por madera acreditando con ello que el encolado es, al menos, tan resistente como la propia madera. Los resultados 
Tabla 6. Valores de la resistencia de la unión dentada.

\begin{tabular}{|l|c|c|c|}
\cline { 2 - 4 } \multicolumn{1}{c|}{} & \multicolumn{3}{c|}{ Fabricante } \\
\cline { 2 - 4 } \multicolumn{1}{c|}{} & A & B & C \\
\hline Resistencia media a flexión $\mathbf{N} / \mathbf{m m}^{\mathbf{2}}$ & $31,39 \pm 0,23$ & $35,40 \pm 0,24$ & $47,67 \pm 0,27$ \\
\hline Desviación típica N/mm $\mathbf{~}^{2}$ & 3,61 & 5,83 & 8,51 \\
\hline Resistencia característica a flexión $\mathbf{N} / \mathbf{m m}^{\mathbf{2}}$ & $28,52 \pm 0,20$ & $29,44 \pm 0,21$ & $35,50 \pm 0,24$ \\
\hline Valor válido para: & GL24 & GL24 & GL28 \\
\hline
\end{tabular}

medios fueron un $70 \%$ para el expediente A, un $90 \%$ para el B y un $80 \%$ para el C.

El análisis de los resultados medios de años anteriores permite conocer la trayectoria del producto fabricado. La resistencia a la flexión respecto a otros expedientes ha disminuido en $16 \mathrm{~N} / \mathrm{mm}^{2}$ de media, sin embargo los valores de fallo por madera se han mantenido similares, de lo que se deduce que el encolado de la unión mantiene sus características adecuadas, pudiendo deberse la reducción en la resistencia a una disminución de la calidad resistente de las piezas de madera que la constituyen. Sin embargo, se deriva del conjunto de los valores obtenidos el cumplimiento del producto para fines estructurales dentro de la clase resistente GL24.

La obtención de un perfil de resistencia elevado no depende sólo de la calidad del encolado sino, también, de la resistencia de la propia madera. Los elementos estructurales sobre los que graviten altas demandas de resistencia deberán ser fabricados con maderas clasificadas mecánicamente y asignadas a clases de resistencia muy elevadas.

\subsection{Madera contralaminada estructural}

Este producto está formado por láminas de madera aserrada empalmadas en sentido longitudinal encoladas a otras dispuestas $90^{\circ}$ respecto a ellas. La constitución recuerda al tablero contrachapado pero incorporando tablas de madera maciza en vez de chapas. Al igual que en los tableros contrachapados, los contralaminados pueden estar integrados por varias capas. El material así consigue altas prestaciones estructurales en las dimensiones deseadas.

Es importante no confundir estos tableros con los utilizados en carpintería o SWP los cuales no sólo están fabricados con piezas de menor tamaño sino que, además, tienen funciones y perfiles resistentes totalmente distintos.

Las aplicaciones de los tableros contralminados son diversas. Se usan fundamentalmente en la construcción de forjados y muros tanto de viviendas unifamiliares como de bloques de pisos o de edificios de oficinas, en la rehabilitación de forjados o como estructuras de cubierta. Posee además unas buenas propiedades para el aislamiento térmico y acústico y resulta sencilla su instalación frente a otros materiales.

La fabricación de estos paneles, al igual que la madera laminada, el producto anterior, obliga al encolado entre capas y al longitudinal entre láminas, por eso una parte importante de la evaluación de sus prestaciones se basa en su valoración.

\subsubsection{Evaluación de la unión dentada en madera contralaminada}

Los expedientes evaluados en el transcurso del último año constaron de 54 muestras. Sobre ellas se realizaron ensayos de flexión de la unión dentada para valoración de la resistencia. Los resultados obtenidos por fabricante se presentan en la Tabla 7 observando que cumplen con los valores de clase de resistencia habituales en este tipo de producto.

La evaluación del resultado requiere adicionalmente analizar el porcentaje de fallo producido en la madera tras la rotura. El valor medio alcanzado para los tres expedientes fue un 85 $\%$ con una desviación típica del $5 \%$, es decir, la resistencia de la unión encolada fue la adecuada ya que en la mayor parte de la sección la rotura se produce antes por el material que por la cola.

Para ver la trayectoria de las características del producto, se compara con los resultados medios por fabricante del año anterior (Figura 6). La resistencia, en términos generales, no manifiesta diferencias significativas en los resultados sin embargo sí se aprecia una ligera disminución en dos de los lotes. El porcentaje de fallo por madera se mantiene en valores para un fabricante mientras que en el otro disminuye. Las causas de la variación de la resistencia pueden ser las mismas que las producidas en el material destinado a madera laminada encolada y que se comentaron en el apartado anterior. De nuevo, los controles periódicos establecidos, permitirán observar la evolución de las propiedades en partidas posteriores.

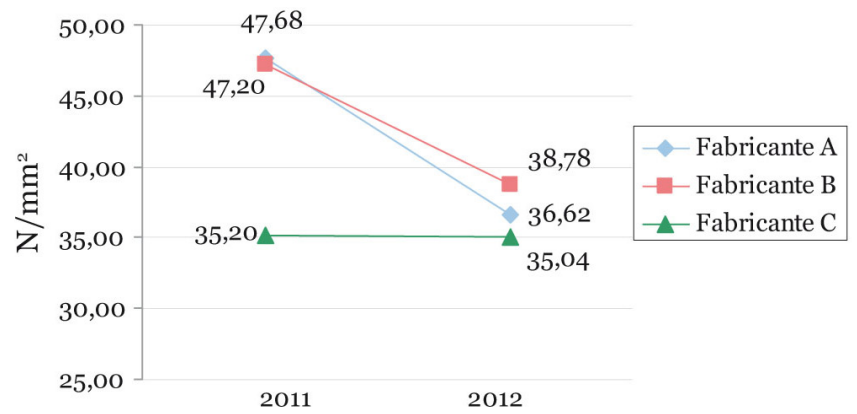

Figura 6. Valores medios de la resistencia a la flexión de la unión dentada.

Tabla 7. Valores medios del ensayo a flexión de la unión dentada.

\begin{tabular}{|l|c|c|c|}
\cline { 2 - 4 } \multicolumn{1}{c|}{} & \multicolumn{3}{c|}{ Fabricantes } \\
\cline { 2 - 4 } \multicolumn{1}{c|}{} & $\mathbf{A}$ & $\mathbf{B}$ & $\mathbf{C}$ \\
\hline Resistencia a flexión $\mathbf{N} / \mathbf{m m}^{\mathbf{2}}$ & $36,62 \pm 0,23$ & $38,78 \pm 0,25$ & $35,04 \pm 0,26$ \\
\hline Desviación típica $\mathbf{N} / \mathbf{m m}^{\mathbf{2}}$ & 6,69 & 9,37 & 8,79 \\
\hline
\end{tabular}




\subsubsection{Valoración de la resistencia al esfuerzo cortante}

Para esta evaluación se utilizaron 135 líneas de cola de un total de 9 muestras. El ensayo se dirige a la determinación de la calidad de la línea de encolado en sentido transversal a la pieza tras haber sido aplicado un ciclo de envejecimiento en función de la clase de uso del material (14). Posteriormente se somete cada línea de cola a un esfuerzo cortante para determinar su resistencia (15) (Figura 7).

Los resultados de varios años para distintos fabricantes se exponen en la Figura 8. La trayectoria de los valores obtenidos dentro del mismo expediente no presenta diferencias significativas notables, siendo el fabricante A el que produce los datos más elevados. Todos los productos analizados cum-

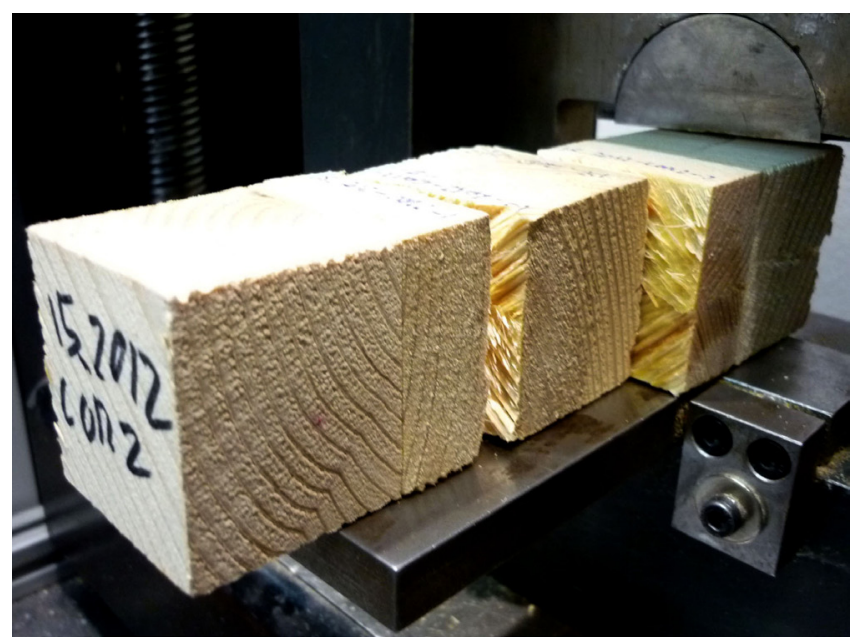

Figura 7. Ensayo de esfuerzo cortante de probetas contralaminadas.

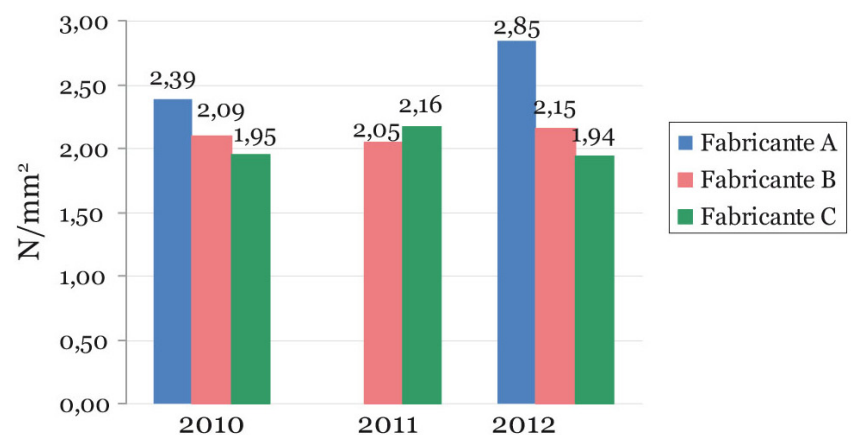

Figura 8. Valores medios de la resistencia al esfuerzo cortante. plen lo especificado en normativa, donde se establece que el $5^{\circ}$ percentil debe ser mayor o igual a $0,8 \mathrm{~N} / \mathrm{mm}^{2}$.

La valoración de la resistencia al cortante de la línea de adhesivo requiere también del análisis del porcentaje de fallo por madera de la unión. Los resultados totales aportan valores entorno al $75 \%$ de media, manteniéndose respecto a evaluaciones anteriores e indicando una correcta unión entre las piezas de madera, al ser mayor que lo establecido como mínimo en normativa (40 \%).

Los tableros contralaminados evaluados presentan valores medios de resistencia y calidad de encolado conformes a las exigencias normativas. Por dicho motivo son productos muy adecuados para ser usados en elementos resistentes tipo placa, siempre y cuando no se superen los umbrales de la clase de servicio 2 (uso interior protegido). Por tanto tienen un amplio elenco de usos entre los que es necesario citar, muy especialmente, la construcción de forjados y muros tanto en obra nueva como en rehabilitación. Debe evitarse sin embargo, su uso completamente exterior ya que las elevadas tensiones que se pueden generar por los acusados cambios en su humedad en servicio pueden generar grietas que sin llegar a arruinar totalmente el material, sí lo afean notablemente.

\section{CONCLUSIONES}

La evaluación de la fabricación y el seguimiento de la calidad de los productos de madera destinados a la construcción ponen de manifiesto su importancia para aportar las garantías reclamadas por el cliente en el momento de la comercialización.

Los ensayos descritos de evaluación de los productos permiten detectar los fallos de los mismos de forma que se podría indicar a las industrias las mejoras encaminadas no solo a solventarlos, sino además a obtener una optimización y rentabilidad superior de ellos.

La necesidad de disponer de equipamiento variado y controlar su mantenimiento, además de personal cualificado, hace que los laboratorios de ensayos debidamente acreditados cumplan una misión imprescindible en el paso de la industria al usuario final. En este sentido, el Laboratorio de Maderas del INIA-CIFOR colabora con la industria para el buen desarrollo y aseguramiento de la calidad de los productos de madera utilizados en construcción.

\section{REFERENCIAS}

(1) DO. (2011). Reglamento (UE) NO 305/2011 del Parlamento Europeo y del Consejo de 9 de Marzo de 2011. Diario Oficial de la Unión Europea, L88, pp. 5-43.

(2) CTE. (2006). Código Técnico de la Edificación. Ministerio de Fomento.

(3) Ministerio de Fomento. (1999, 6 de noviembre). LOE - Ley de Ordenación de la Edificación. Boletín Oficial del Estado, $\mathrm{n}^{0}$ 266, pp. 38925-38934.

(4) AENOR. (2006). UNE-EN 789:2006. Estructuras de madera. Métodos de ensayo. Determinación de las propiedades mecánicas de los tableros derivados de la madera. Asociación Española de Normalización.

(5) Peraza-Sánchez, F., Arriaga-Martitegui, F., Peraza-Sánchez, J.E. (2004). Tableros de madera de uso estructural. Madrid: AITIM.

(6) AENOR. (2001). UNE-EN 12369-1:2001. Tableros derivados de la madera. Valores característicos para el cálculo estructural. Parte 1: OSB, tableros de partículas y tableros de fibras. Asociación Española de Normalización. 
(7) AENOR. (2006). UNE-EN 13986:2006. Tableros derivados de la madera para utilización en la construcción. Características de evaluación de la conformidad y marcado. Asociación Española de Normalización.

(8) AENOR. (1994). UNE-EN 323: 1994. Tableros derivados de la madera. Determinación de la densidad. (Versión oficial EN 323:1993). Asociación Española de Normalización.

(9) Peraza-Sánchez, F., Peraza Sánchez, J.E. (2010). Guía de la Madera (I). Productos básicos y carpintería. Madrid: AITIM.

(10) Ahvenainen, J., García-Barbero, M. (2007). Uso estructural de vigas Dúo y Trío. Vigas de madera compuestas por dos o tres piezas: una alternativa ideal para estructuras ligeras de alta exigencia estética. Nota técnica. Informes de la Construcción, 59(506): 117-121.

(11) AENOR. (2002). UNE-EN 386:2002. Madera laminada encolada. Especificaciones y requisitos de fabricación. Asociación Española de Normalización.

(12) AENOR. (2012). UNE-EN 408:2011+A1:2012. Estructuras de madera. Madera aserrada y madera laminada encolada para uso estructural. Determinación de algunas propiedades físicas y mecánicas. Asociación Española de Normalización.

(13) AENOR. (2010). UNE-EN 14080:2006. ERRATUM 2010. Estructuras de madera. Madera laminada encolada. Requisitos. Asociación Española de Normalización.

(14) AENOR. (2009). UNE-EN 13354: 2009. Tableros de madera maciza. Calidad de encolado. Método de ensayo. Asociación Española de Normalización.

(15) AENOR. (1995). UNE-EN 392: 1995. Madera laminada encolada. Ensayo de esfuerzo cortante en líneas de adhesivo. Asociación Española de Normalización. 